\title{
Creencias y prácticas alimentarias e higiénicas en madres de niños menores de 5 años en el distrito de Anta, Áncash, Perú
}

Beliefs and practices about food and hygiene in mothers of children under 5 years in Anta district, Ancash, Peru

Gina Mendoza RamíreZ ${ }^{1}$

\section{RESUMEN}

Una nutrición adecuada, en cantidad y calidad, es clave para el desarrollo físico e intelectual del niño. Un niño que sufre desnutrición ve afectada su supervivencia y el buen desarrollo de su cuerpo y de sus capacidades cognitivas e intelectuales. Por ello se realizó un estudio descriptivo, con una muestra de 290 niños menores de 5 años y su respectiva madre, con el objetivo de determinar la asociación entre las creencias y prácticas alimentarias e higiénicas de las madres con el estado nutricional de sus hijos menores de 5 años, en el distrito de Anta (Áncash). Los resultados muestran que, 4 de cada 10 niños menores de cinco años sufre de desnutrición crónica. Creencias perjudiciales como el inicio precoz de la alimentación complementaria, y que este inicio debe hacerse con alimentos ligeros fueron asociadas a la desnutrición crónica. El iniciar la alimentación complementaria antes de los 6 meses, consumir sólo 2 comidas principales al día en lugar de 3 y consumir alimentos disminuidos en frecuencia, consistencia y cantidad durante los procesos infecciosos son prácticas asociadas a la aparición de desnutrición crónica, así como la eliminación de excretas al campo abierto en lugar de usar inodoro o letrina.

Palabras clave: desnutrición; creencias; prácticas alimentarias.

\begin{abstract}
Proper nutrition, in quantity and quality, is key to the physical and intellectual development of child. A child suffering from inadequate nutrition affected their survival and proper development of their body and their cognitive and intellectual
\end{abstract}

1 Universidad Nacional «Santiago Antúnez de Mayolo». Huaraz, Perú. 
abilities. Thus, a descriptive study was conducted with a sample of 290 children under 5 years and their respective mother, in order to determine the association between beliefs and food and hygiene practices of mothers with the nutritional status of their minor children 5 years in the district of Anta (Ancash). The results show that 4 out of 10 children under five suffer from chronic malnutrition. Harmful beliefs as children of 4 or 5 months can already start their complementary feeding and that this start should be made with light foods were associated with chronic malnutrition. The start complementary feeding before 6 months, consuming only 2 main meals a day instead

of 3 and consume decreased in frequency, consistency and quantity during infectious processes foods are practices associated with the occurrence of chronic malnutrition, as well as the elimination excreta to open instead of using toilet or latrine field.

Keywords: malnutrition; beliefs; dietary practices.

\section{ICHIKLLACHAW}

Atska mikuykunawan alli kaq mikuy kayninqa wamrakunapa kurkunkunatawan tuqshunkuna alli wiñayaananpaqmi. Huk wamra mana alli mikukuqqa kawayninchaw, wiñaynichaw, hina tuqshun puquyninpaqmi allitsuqa. Tsayraykurmi kay musyapakuy imanaw kaqpaq willakuykunata rurakashqa, kay musyapakuyqa 290 manaraq 5 wataman chaqñushpi wamrakunachawmiuryakashqa. Tsayllapanchawimanawmi iñinninkunawan mamakunapa mikukuyninkuna tinkuynin kanqantam tsuyantsaatsimun. Kay musyapakuyqa Anta ichik llaqtachawmi rurakashqa (anqashchaw). Kaychaw tarikashqa 10 wamrakunapiq 4kum patsaypa mana alli mikuyan. Lluta iñiyninkunaraykurmi, paykuna llutam kaynaw rimayan ñushpikunaqa ichik ichikllam mikuyanman niyanmi. Manaraq 6 killaman char 2 alli mikuyllatam huk hunaqchaw mikuyanman, manam 3 kutiqa mikuyanmantsu, tsayraykurmi uyuyaymanpis, qishyaymanpis tikrayan; hinamanpis haqchaw ismayanqanraykur tsayman tikrayan, mana tsaynaw kananpaqqa ismakuna wayichawchi alliqa ismakuyanman.

Pushaq shimikuna: mana alli mikukuy; uyuyay; iñiykuna; alli mikukuykuna.

\section{INTRODUCCIÓN}

Los requerimientos de energía, proteína y micronutrientes específicos necesarios para el buen funcionamiento del organismo, se satisfacen a partir de la cantidad y variedad de los alimentos ingeridos. Por ello, la calidad de la dieta cotidiana constituye un elemento esencial que contribuye al estado de nutrición y salud del individuo, especialmente en los primeros años de vida. La malnutrición por defecto, o desnutrición, es calificada como una emergencia silenciosa, ya que está directamente relacionada con la 
mitad de los casos de mortalidad infantil en el mundo, ocupa uno de los primeros lugares de mortalidad, y sus principales víctimas son los niños menores de 5 años (FAO, 2012). En lo que se refiere a desnutrición es importante señalar que existen tres tipos: desnutrición aguda o emaciación, desnutrición global o insuficiencia ponderal y desnutrición crónica o talla baja. Esta última no se traduce solo en un problema de salud, sino también en una limitante para el desarrollo del país (Cortez, 2002). La desnutrición crónica infantil se asocia a daños físicos y cognitivos de difícil reversión, lo que afecta la salud del niño en el presente con efectos en su bienestar social y productividad económica en el futuro. Las consecuencias de una nutrición deficiente continúan en la edad adulta y afectan a la próxima generación, debido a que las niñas y mujeres desnutridas tienen mayor riesgo de tener hijos desnutridos (UNICEF, 2008).

En los últimos años, la investigación en el campo del crecimiento y desarrollo infantil ha sido intensa y extensa. Uno de sus objetivos es la identificación de patrones y determinantes del crecimiento por ser un indicador de salud infantil, desarrollo poblacional y en consecuencia un elemento central de atención en Salud Pública (FAO, 2012). En países en desarrollo como el Perú, la insatisfacción crónica de necesidades básicas condiciona negativamente tanto el crecimiento como el desarrollo en la mayoría de los niños (Lutter y Chaparro, 2008). Más allá de la precariedad del ingreso económico, son múltiples las vías mediante las cuales los ambientes pluricarenciales e insalubres perjudican el estado nutricional y el crecimiento. La alta morbilidad y mortalidad infantil en este contexto, implica importantes costos en bienestar, vidas y recursos, actuando de fondo errores cotidianos, alimentarios e higiénicos, que en principio se puede corregir. Variables como la selección de alimentos, la dedicación de la madre, las actitudes y prácticas alimentarias e higiénicas en el hogar, participan activamente en la diaria alimentación y nutrición de los niños. El hecho de que el problema, aun siendo de naturaleza multicausal sea predominantemente prevenible, conmina a los actores del sector salud a desarrollar estrategias no sólo en el campo terapéutico o curativo sino también en el área preventiva. Se considera que el recurso preventivo y correctivo más eficaz, duradero y menos costoso es la orientación alimentaria. No obstante, intentos serios en la modificación de hábitos en el ámbito individual o poblacional con claros objetivos preventivos muestran éxitos parciales o temporales, destacándose la complejidad del proceso de transformación sostenible en el área de hábitos alimentarios y/o higiénicos (Urteaga y Pinheiro, 2003). Por ello, se considera importante la comprensión de los elementos que condicionan el comportamiento materno en el área, desde los que influyen en la aceptación de propuestas educativas, hasta los relacionados directamente con la adquisición y mantenimiento de hábitos saludables. La conducta materna en el área alimentaria e higiénica es decisiva en la salud del niño y puede depender de factores externos como la disponibilidad y acceso a los alimentos, pero también de recursos internos que afectan el comportamiento, como son sus creencias, nivel educativo y pa- 
trones culturales tanto individuales como comunitarios (Huamán, 2007). La transformación del conocimiento en acción o conducta, se relaciona con ambos. En el medio rural, frecuentemente en un contexto de similitudes, coexisten niños eutróficos con desnutridos; niños con y sin infecciones gastrointestinales, lo cual sugiere la posibilidad de que existan factores, generados por las madres, de efecto protector o por el contrario perjudicial para el estado de salud y nutrición de individuos vulnerables (Paraje, 2008). Por ello, la presente investigación respondió a la siguiente pregunta: ¿Cuáles son las creencias y prácticas alimentarias e higiénicas de las madres de niños menores de 5 años, distrito de Anta, y cuál es su relación con el estado nutricional de sus hijos?, lo que permitió identificar entre patrones referenciales y conductuales maternos, hábitos protectores reproducibles o perjudiciales para su señalamiento y prevención, a fin de optimizar el estado nutricional y crecimiento de niños en ambientes de pobreza y pobreza extrema.

\section{MATERIALES Y MÉTODOS}

Se realizó una investigación aplicada que genera conocimiento sobre las creencias y prácticas alimentarias para la aplicación directa de intervenciones en los niños del distrito de Anta (Áncash). De acuerdo al nivel de profundidad es un estudio descriptivo analítico.

Se tomó como unidad de análisis a un menor de 5 años con su respectiva madre, los que conformaron la diada de estudio. Para una población pequeña como ésta, buscando la máxima representatividad se trabajó con el total de la población. Así, la muestra se conformó con 290 niños menores de 5 años y sus madres, residentes en el distrito de Anta, durante el año 2009.

La recolección de datos se hizo a través de una encuesta validada con un error de medida del 5\%. La aplicación se realizó en entrevista directa. La evaluación nutricional antropométrica se determinó midiendo el peso y la talla de los niños en una balanza y un tallímetro (previamente calibrados, con un error de medida de +-20 gr. y +-0.5 $\mathrm{cm}$.). Se estudió el comportamiento del puntaje $\mathrm{Z}$ en los indicadores antropométricos de talla para la edad (T/E), peso para la edad (P/E) y peso para la talla $(\mathrm{P} / \mathrm{T})$, tomando como base el Patrón de Crecimiento Infantil de la Organización Mundial de la Salud, y utilizando como punto de corte los indicadores $(\mathrm{T} / \mathrm{E}),(\mathrm{P} / \mathrm{E})$ y $(\mathrm{P} / \mathrm{T})$ por debajo de 2 desviaciones estándar.

Los datos cuantitativos fueron procesados mediante el programa estadístico SPSS versión 17.0. Para determinar si existe asociación entre las variables de estudio se utilizó la diferencia no paramétrica con la prueba chi cuadrado por tratarse de variables cualitativas. Este análisis se realizó teniendo en cuenta un nivel de significación del 5\% 
$(\mathrm{p}<0,05)$. Así mismo se calculó el Ods ratio con un intervalo de confianza del 95\%, para medir la fuerza de la asociación entre las variables de estudio.

\section{RESULTADOS}

1. Estado nutricional de niños menores de 5 años

Se encontró mayoritariamente malnutrición por déficit, especialmente desnutrición crónica $(38,3 \%)$ y desnutrición global (12,8\%), es decir, niños con 2 o más desviaciones estándar (DE) por debajo de la media del patrón internacional utilizado por la OMS para los indicadores de talla/edad y peso/talla respectivamente.

Con relación a la desnutrición en menores de 5 años, el indicador talla/edad es el que menores valores tuvo en los distintos grupos de edad, evidenciándose que a mayor edad mayor es la desnutrición crónica que presentan los niños. La desnutrición global, que no tiene presencia entre los menores de 6 meses fue incrementándose hasta alcanzar su pico de presentación entre los niños de 1 año (29,4\%), para luego descender hasta 5,2\% entre los niños de 4 años; en oposición a lo que ocurre con la desnutrición crónica que afectó al 41,2\% de los niños de 1 año, alcanzando al 57,6\% de los de 3 años y al 51,7\% de los niños de 4 años. La desnutrición aguda fue la menos prevalente, pues afecta sólo al 3,1\% de los niños de 6 a 11 meses (figura 1).

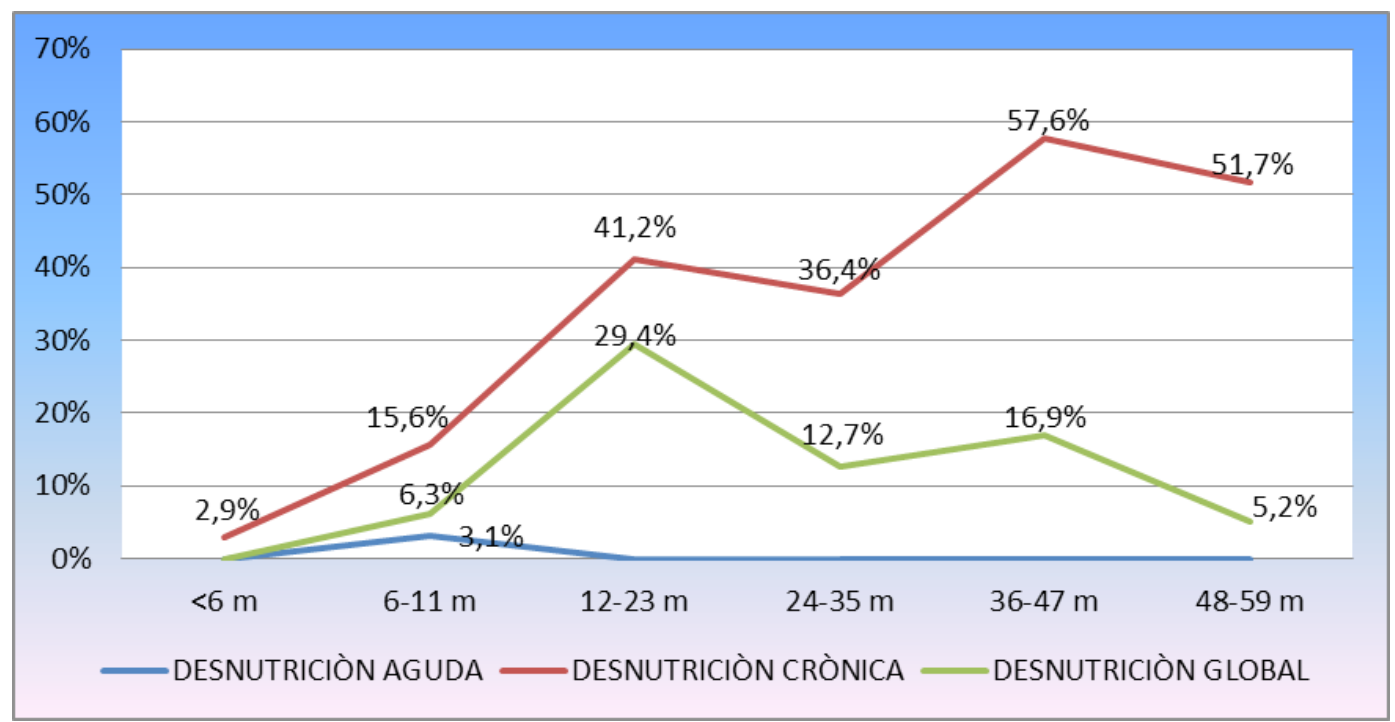

Figura 1. Desnutrición según grupos de edad en menores de 5 años del distrito de Anta 
2. Creencias sobre alimentación e higiene

Las principales creencias sobre alimentación e higiene que poseen las madres se resumen en la tabla 1, destacando que gran parte de ellas muestran diferencias estadísticamente significativas, pues el valor $\mathrm{P}$ del estadígrafo chi cuadrado, resultó menor de 0,05 .

Tabla 1. Creencias de las madres sobre alimentación e higiene de niños menores de 5 años del distrito de Anta

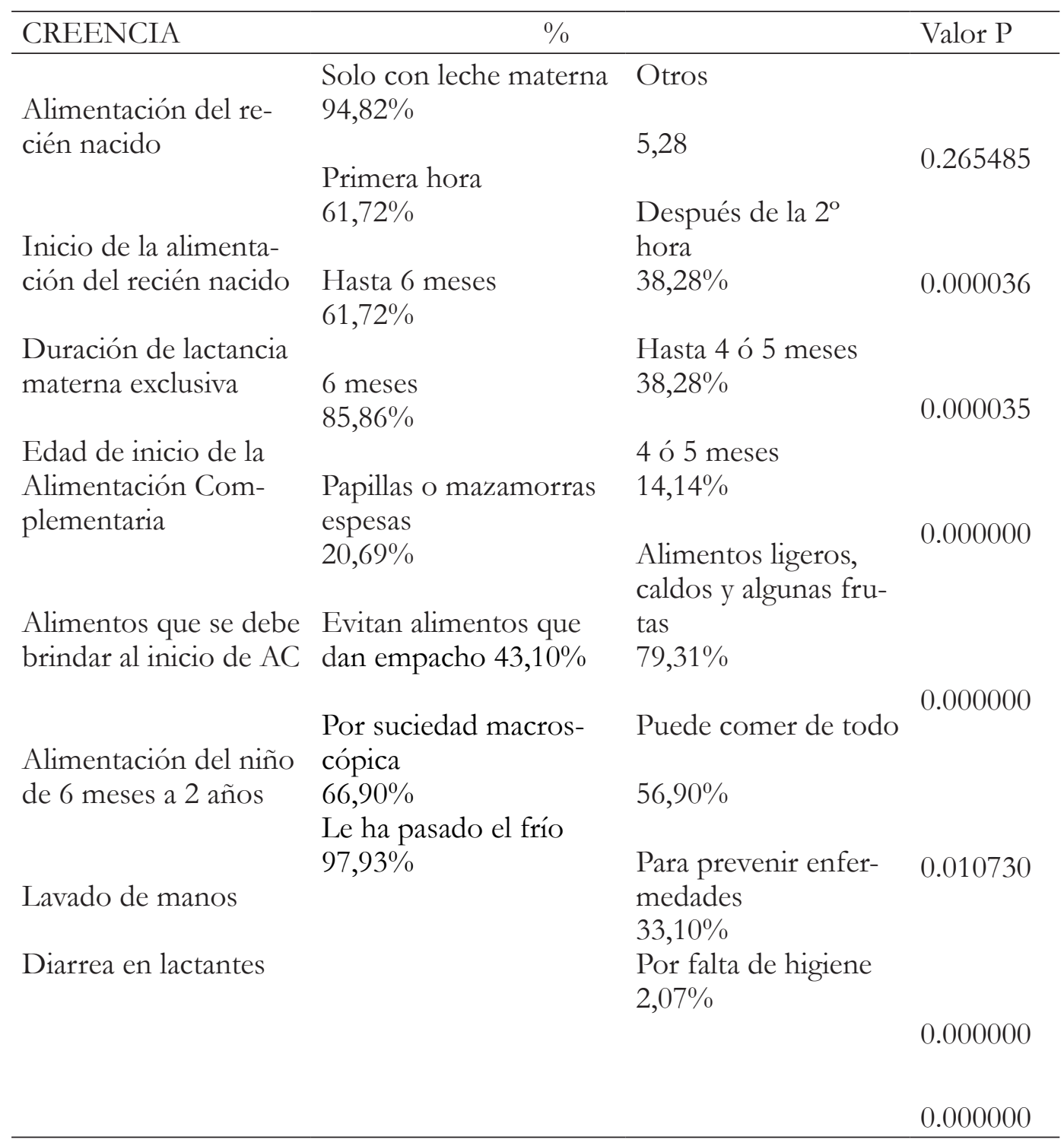


3. Prácticas alimentarias e higiénicas

Tabla 2. Prácticas alimentarias e higiénicas de madres de niños menores de 5 años del distrito de Anta

\begin{tabular}{|c|c|c|c|}
\hline PRÁCTICA & $\%$ & $\%$ & $\%$ \\
\hline & \multicolumn{3}{|c|}{ Lactancia materna } \\
\hline $\begin{array}{l}\text { Inicio de alimentación } \\
\text { del recién nacido }\end{array}$ & $\begin{array}{c}\text { Durante la } 1^{\circ} \\
\text { hora } \\
54,8 \%\end{array}$ & $\begin{array}{c}\text { Entre } 2^{\circ} \text { y } 6^{\circ} \\
\text { hora } \\
25,5 \%\end{array}$ & $\begin{array}{c}\text { Después de la } 6^{\circ} \\
\text { hora } \\
19,7 \%\end{array}$ \\
\hline $\begin{array}{l}\text { Alimentación del menor } \\
\text { de } 6 \text { meses }\end{array}$ & $\begin{array}{l}\text { Lactancia mater- } \\
\text { na exclusiva } \\
58,4 \%\end{array}$ & $\begin{array}{l}\text { Lactancia mater- } \\
\text { na predominante } \\
27,1 \%\end{array}$ & $\begin{array}{l}\text { Lactancia mixta } \\
\qquad 14,5 \%\end{array}$ \\
\hline $\begin{array}{l}\text { Lactancia Materna Am- } \\
\text { pliada }\end{array}$ & $\begin{array}{c}\text { Entre } 12 \text { y } 17 \\
\text { meses } \\
27,8 \%\end{array}$ & $\begin{array}{c}\text { Entre } 18 \text { y } 23 \\
\text { meses } \\
30,9 \%\end{array}$ & $\begin{array}{c}24 \text { meses o más } \\
41,3 \%\end{array}$ \\
\hline $\begin{array}{l}\text { Edad de inicio de Ali- } \\
\text { mentación Complemen- } \\
\text { taria }\end{array}$ & 6 meses & 4 ó 5 meses & Más de 6 meses \\
\hline Diversidad de la dieta & $\begin{array}{c}65,1 \% \\
3 \text { grupos básicos } \\
20,4 \%\end{array}$ & $\begin{array}{c}32,2 \% \\
\text { Sólo calóricos } \\
43,5 \%\end{array}$ & $\begin{array}{c}2,7 \% \\
\text { Sin reguladores } \\
36,1 \%\end{array}$ \\
\hline \multirow{2}{*}{$\begin{array}{l}\text { Frecuencia } \\
\text { de alimentación del niño } \\
\text { menor de } 5 \text { años }\end{array}$} & $\begin{array}{l}\text { Adecuada } \\
42,4 \%\end{array}$ & $\begin{array}{c}\text { Insuficiente } \\
57,6 \%\end{array}$ & $\begin{array}{c}\text { En exceso } \\
0 \%\end{array}$ \\
\hline & Correcto & Incorrecto & \\
\hline Lavado de manos & $47,2 \%$ & $52,8 \%$ & \\
\hline Eliminación de excretas & $\begin{array}{c}\text { Desagüe o letrina } \\
33,4 \%\end{array}$ & $\begin{array}{l}\text { Letrina } \\
32,1 \%\end{array}$ & $\begin{array}{c}\text { Campo abierto } \\
34,5 \%\end{array}$ \\
\hline
\end{tabular}

4. Creencias y desnutrición

4.1. Creencia sobre edad de inicio de la alimentación complementaria y desnutrición crónica

La tabla 3 muestra las diferencias estadísticamente significativas entre las creencias de las madres de niños con desnutrición y sin ella, lo que nos permite aceptar la hipótesis alterna de investigación, y afirmar que la creencia inicio de alimentación complementaria antes de los 6 meses es un factor que se relaciona a la presencia de desnutrición crónica en los niños menores de 5 años. 
Tabla 3. Creencia sobre edad de inicio de la alimentación complementaria y desnutrición crónica de niños menores de 5 años del distrito de Anta

\begin{tabular}{cccc}
\hline $\begin{array}{c}\text { Creencia sobre edad } \\
\text { de inicio }\end{array}$ & $\begin{array}{c}\text { Niños con Desnutri- } \\
\text { ción crónica }\end{array}$ & $\begin{array}{c}\text { Niños sin desnutri- } \\
\text { ción crónica }\end{array}$ & Total \\
\hline $4-5$ meses & $55,8 \%$ & $40,8 \%$ & $46,6 \%$ \\
6 meses & $44,2 \%$ & $59,2 \%$ & $65,1 \%$ \\
Total & $100 \%$ & $100 \%$ & $100 \%$ \\
\hline & $\mathrm{x}^{2}=4,822$ & $\mathrm{p}=0,028$
\end{tabular}

\subsection{Creencias sobre consistencia de alimentación y desnutrición crónica}

Las diferencias existentes entre las creencias de las madres sobre la consistencia de la alimentación complementaria fueron estadísticamente significativas $(p<0,05)$ con un intervalo de confianza de 2,586 - 10,922; por tanto, se acepta la hipótesis alterna y se puede afirmar que la creencia de que el inicio de la alimentación complementaria debe hacerse con alimentos de consistencia ligera es un factor asociado al desarrollo de desnutrición crónica en los niños menores de 5 años.

Tabla 3. Creencias sobre consistencia de alimentación complementaria y desnutrición crónica de niños menores de 5 años del distrito de Anta

\begin{tabular}{cccc}
\hline $\begin{array}{c}\text { Consistencia de ali- } \\
\text { mentación comple- } \\
\text { mentaria }\end{array}$ & $\begin{array}{c}\text { Niños con Desnutri- } \\
\text { ción crónica }\end{array}$ & $\begin{array}{c}\text { Niños sin desnutri- } \\
\text { ción crónica }\end{array}$ & Total \\
\hline $\begin{array}{c}\text { Tipo caldos o maza- } \\
\text { morras ligeras } \\
\text { Purés, papillas o ma- } \\
\text { zamorras espesas } \\
\text { Total }\end{array}$ & $51,3 \%$ & $16,8 \%$ & $30 \%$ \\
\hline & $100 \%$ & $83,2 \%$ & $70 \%$ \\
& $\mathrm{x}^{2}=39,635$ & $\mathrm{p}=0,0001$ & $100 \%$ \\
\hline
\end{tabular}

5. Prácticas alimentarias y desnutrición

\subsection{Edad de inicio de la alimentación complementaria y desnutrición}

El 59\% de los niños con desnutrición crónica inició su alimentación complementaria a una edad inadecuada (antes de los 6 meses o después), frente al 83,5\% de los niños eutróficos que iniciaron su alimentación complementaria a los 6 meses. Estas diferencias resultaron estadísticamente significativas $(\mathrm{p}<0,05)$; por lo que la edad de inicio inadecuada de la alimentación complementaria actúa como un factor de riesgo para que los niños menores de 5 años desarrollen desnutrición crónica. 
Tabla 4. Edad de inicio de la alimentación complementaria y desnutrición crónica de niños menores de 5 años del distrito de Anta

\begin{tabular}{cccc}
\hline Edad de inicio & $\begin{array}{c}\text { Desnutrición cró- } \\
\text { nica }\end{array}$ & $\begin{array}{c}\text { Sin desnutrición } \\
\text { crónica }\end{array}$ & Total \\
\hline $\begin{array}{c}\text { 4-5 meses o más de } \\
6 \text { meses }\end{array}$ & $59 \%$ & $16,5 \%$ & $34,9 \%$ \\
6 meses & $41 \%$ & $83,5 \%$ & $65,1 \%$ \\
Total & $100 \%$ & $100 \%$ & $100 \%$ \\
\hline & $\mathrm{x}^{2}=4,269$ & $\mathrm{p}=0,039$
\end{tabular}

\subsection{Frecuencia de alimentación complementaria y desnutrición crónica}

El 31,8\% de los niños con desnutrición crónica sólo consumió 2 comidas principales el día anterior a la encuesta, mientras que el 90,3\% de los niños que no presentaron desnutrición crónica sí consumió 3 comidas principales. Estas diferencias, que muestran significancia estadística $(\mathrm{p}<0,05)$, muestran también a esta práctica inadecuada de alimentación como un factor de riesgo para que los niños desarrollen desnutrición crónica.

Tabla 5. Frecuencia de alimentación complementaria y desnutrición crónica de niños menores de 5 años del distrito de Anta

\begin{tabular}{cccc}
\hline $\begin{array}{c}\text { Comidas principales } \\
\text { al día }\end{array}$ & $\begin{array}{c}\text { Niños con Desnutri- } \\
\text { ción crónica }\end{array}$ & $\begin{array}{c}\text { Niños sin desnutri- } \\
\text { ción crónica }\end{array}$ & Total \\
\hline 2 & $31,8 \%$ & $9,7 \%$ & $19,2 \%$ \\
3 o más & $67,2 \%$ & $90,3 \%$ & $80,8 \%$ \\
Total & $100 \%$ & $100 \%$ & $100 \%$ \\
\hline $\mathrm{x}^{2}=15,79$ & $\mathrm{p}=0,0000708$ & I.C. $=1,95-9,04$ &
\end{tabular}

\subsection{Alimentación durante la enfermedad y desnutrición crónica}

En la tabla 6 se muestra las diferencias entre la alimentación durante los episodios de enfermedad que recibieron los niños con desnutrición y sin ella, las mismas que resultaron significativas $(\mathrm{p}<0,05)$; lo que permite concluir que una alimentación disminuida en frecuencia, consistencia y cantidad brindada a los niños menores de 5 años durante la enfermedad los pone en mayor riesgo de padecer desnutrición crónica. 
Tabla 6. Alimentación durante la enfermedad y desnutrición crónica de niños menores de 5 años del distrito de Anta

\begin{tabular}{cccc}
\hline $\begin{array}{c}\text { Alimentación duran- } \\
\text { te la enfermedad }\end{array}$ & $\begin{array}{c}\text { Niños con Desnutri- } \\
\text { ción crónica }\end{array}$ & $\begin{array}{c}\text { Niños sin desnutri- } \\
\text { ción crónica }\end{array}$ & Total \\
\hline Disminuye & $51,8 \%$ & $27,6 \%$ & $38 \%$ \\
Igual o más & $48,2 \%$ & $72,4 \%$ & $62 \%$ \\
Total & $100 \%$ & $100 \%$ & $100 \%$ \\
\hline
\end{tabular}

\subsection{Prácticas de higiene y desnutrición}

La eliminación de excretas humanas al campo abierto resultó una práctica de higiene común en el 43,2\% de los niños con desnutrición crónica, mientras que en el 71\% de los niños eutróficos las excretas fueron eliminadas a través de un desagüe o letrina. Estas diferencias resultaron estadísticamente significativas $(p<0.05)$; permitiéndonos aceptar la hipótesis alterna de investigación e infiriendo que los niños que carecen de un sistema adecuado de eliminación de excretas tienen mayor riesgo de presentar desnutrición crónica.

\section{DISCUSIÓN}

La prevalencia de desnutrición crónica entre los niños menores de 5 años encontrada en el presente estudio $(38,3 \%)$ guarda similitud con lo reportado por la ENDES Continua 2009 para la zona rural de Áncash (38,8\%), aunque dicha cifra se encuentra 1,5 puntos porcentuales por debajo de la proporción rural a nivel de todo el Perú (40,3\%); pero muy por encima del promedio regional $(28,2 \%)$ y nacional $(23,8 \%)$, lo que refleja las brechas aún existentes entre la zona rural y urbana. Sin embargo, estos resultados difieren en 6 puntos porcentuales con lo reportado en el INEI (2010) para zonas rurales de la sierra $(45,2 \%)$ pero muestran, una vez más, la estrecha relación entre ruralidad y desnutrición crónica, ya que las cifras de desnutrición crónica, según este mismo estudio, descienden drásticamente a 13,3\% en la zona costa y a 6,7\% en Lima Metropolitana.

Estas cifras de desnutrición crónica, especialmente, hacen pensar no sólo en las causas subyacentes de esta deficiencia nutricional sino también en los efectos negativos que la misma produce en el estado de salud del niño, en su habilidad para aprender, para comunicarse, para desarrollar el pensamiento analítico, la socialización y la habilidad de adaptarse a nuevos ambientes. Por tanto, desde un punto de vista social, las consecuencias de la desnutrición son graves. El daño que se genera en el desarrollo cognitivo de 
los individuos afecta el desempeño escolar, en un primer momento y, más adelante, la capacidad productiva laboral. Peor aún, una niña desnutrida tiene mayor probabilidad de procrear, cuando sea adulta, a un niño desnutrido, perpetuando los daños de generación en generación (Lutter y Chaparro, 2008).

Las prácticas de alimentación de las madres de niños menores de 5 años son, en su mayoría, inadecuadas; datos comparables con los encontrados por Huamán (2007) en pobladores de pobreza y pobreza extrema del Perú (6 meses y 57,5\% respectivamente) y los reportados por el INEI (2010) (60,2\% de lactancia materna exclusiva para Áncash), pero inferior al promedio nacional, que según esta fuente, alcanza el 69,9\% de lactancia materna exclusiva y se encuentran entre los más altos de América Latina. Estas cifras, nos indican que sólo 6 de cada 10 niños menores de 5 años recibió lactancia materna exclusiva y los otros cuatro recibieron otras leches, o peor aún, otros líquidos o alimentos de baja densidad energética, los mismos que según Kuklina y otros (2004) desplazan a la leche materna y a los nutrientes altamente bio-disponibles que provee, contribuyendo a la desnutrición crónica e incrementando el riesgo a padecer infecciones diarreicas. Estas cifras de incumplimiento de lactancia materna exclusiva guardan estrecha relación con las creencias que las madres tienen acerca de su duración, ya que, si bien es cierto, gran porcentaje de ellas $(71,72 \%)$ la identifican como una medida adecuada para asegurar el crecimiento de sus hijos, muchas de ellas no creen que exclusivamente deba darse leche materna hasta los 6 meses. Zarzalejo y otros (2001) reportaron que 53\% de los niños que asistieron al Centro de Atención Nutricional Infantil Antímano en Venezuela recibieron alimentos complementarios antes de los 4 meses. Así mismo, el Ministerio de Salud de Guatemala, reportó el frecuente uso de biberón entre los menores de 6 meses, la administración de líquidos y el inicio temprano de la alimentación complementaria, motivado por la inseguridad de las madres en cuanto a la calidad y cantidad de la leche producida por ellas, y la interpretación de algunos gestos del niño como deseo de comida (Alvarez y Gonzales, 2002).

Si bien los Lineamientos de Nutrición Materno Infantil del Perú establecidos por el Instituto Nacional de Salud (2004) establecen que la alimentación del niño mayor de 6 meses debe estar constituida por 3 comidas principales y 2 entre comidas, conforme crece; y estos contenidos forman parte de la consejería que brinda el personal de salud a las madres de niños menores de 5 años, muchas veces se quedan en conocimientos y no son llevados a la práctica. Es así que, el 31,8\% de los niños con desnutrición crónica sólo consumió 2 comidas principales el día anterior a la encuesta, mientras que el 90,3\% de los niños que no presentaron desnutrición crónica sí consumió 3 comidas principales. Esta práctica de alimentación, que es común en la zona rural de nuestro país, como lo mencionara Huamán (2007), tiene su origen en la inseguridad alimentaria de su población, que vive en pobreza extrema. Dedicados a la agricultura y ganadería 
de subsistencia, tienen como práctica cotidiana el consumo de un desayuno abundante constituido principalmente por una sopa de harina de trigo, habas o habichuelas, acompañada de papas sancochadas o cancha; para luego trasladarse a sus labores de pastoreo o labrado de la tierra hasta las 5 de la tarde, hora en que consumen su segunda comida principal con características similares a las del desayuno (CEPAL, 2007).

La falta de servicios de saneamiento constituye un entorno doméstico poco saludable por la posibilidad de infecciones, especialmente diarreas y parasitosis, la que a su vez es causa inmediata de desnutrición (Horodynski y Stommel, 2005). La eliminación de excretas humanas al campo abierto resultó una práctica de higiene común en el 43,2\% de los niños con desnutrición crónica de nuestro estudio, mientras que en el $71 \%$ de los niños eutróficos las excretas fueron eliminadas a través de un desagüe o letrina. Estas diferencias estadísticamente significativas y su intervalo de confianza muestran que los niños que carecen de un sistema adecuado de eliminación de excretas tienen más riesgo de presentar desnutrición crónica. Cifras altas de inaccesibilidad a servicios de saneamiento básico son un común denominador en las zonas rurales, como lo reporta el INEI (2010), 33,6\% a nivel nacional y 38\% en Áncash; aunque es importante resaltar que ha disminuido en 16,1 y 23,3 puntos porcentuales respectivamente en relación al año 2000.

\section{CONCLUSIONES}

La desnutrición crónica infantil en el distrito de Anta es un problema grave. 4 de cada 10 niños menores de cinco años sufren de este mal. Se identificaron creencias perjudiciales para la salud y nutrición de los niños menores de 5 años como: el inicio temprano de la alimentación complementaria, la misma que debe hacerse con alimentos de baja densidad energética como caldos y sopas; la exclusión de algunos alimentos como las menestras de la dieta de los menores de 1 año, y la atribución, como causas de diarrea, a la exposición de la madre que da de lactar, al frío, o el consumo por parte de la misma de menestras o comida calentada, más no a falta de medidas higiénicas.

Las prácticas alimentarias resultaron, frecuentemente, inadecuadas: abandono de lactancia materna exclusiva e inicio precoz de la alimentación complementaria; dieta eminentemente rica sólo en carbohidratos y de consistencia líquida o semilíquida; consumo de sólo 2 comidas principales al día; frecuencia, consistencia y cantidad disminuida de los alimentos durante la enfermedad y ausencia de una comida adicional en las dos semanas posteriores a la misma. Así mismo, prácticas inadecuadas de lavado de manos fueron reportadas por la mitad de las madres del estudio y la eliminación de excretas al campo abierto por casi 4 de cada 10 madres.

La creencia de que los niños menores de 5 meses ya pueden iniciar su alimentación 
complementaria, y que este inicio debe hacerse con alimentos de baja densidad energética como caldos y sopas, están asociadas a la desnutrición crónica. El iniciar la alimentación complementaria antes de los 6 meses, consumir sólo 2 comidas principales al día en lugar de 3 y consumir alimentos disminuidos en frecuencia, consistencia y cantidad durante los procesos infecciosos son prácticas asociadas a la aparición de desnutrición crónica, así como la eliminación de excretas al campo abierto en lugar de usar inodoro o letrina.

\section{REFERENCIAS BIBLIOGRÁFICAS}

Álvarez, Martha y González, Laura. 2002. «Prácticas alimentarias en las familias del área rural de Medellín-Colombia». ALAN. Caracas. Vol. 52, No 1. 55-62.

Comisión Económica para América Latina y el Caribe. 2007. El costo Social del Hambre: Impacto Social de la Desnutrición Infantil en el Perú. Lima.

Cortez, Rafael. 2002. La nutrición de los niños en edad preescolar. Lima: Centro de Investigación de la Universidad del Pacífico.

FAO. 2012. Panorama de la Seguridad Alimentaria y Nutricional de América Latina y el Caribe. Santiago.

Horodynski, Mildred y Stommel, Manfred. 2005. «Nutrition Education Aimed at Toddlers: An Intervention Study». Pediatric Nursing. Vol. 31, No 5.364 - 372.

Huamán, Lucio. 2007. Estado Nutricional y Prácticas Alimentarias de la Población Infantil Menor de 5 Años en Distritos en Pobreza Extrema en Perú. Lima: INS.

INEI. 2010. Perú: Encuesta Demográfica y de Salud Familiar-ENDES Continua. Lima.

INS-CENAN. 2004. Lineamientos de Nutrición Materno Infantil del Perú. Lima: MINSA-INS.

Kuklina, Elena y otros. 2004. «Growth and Diet Quality Are Associated with the Attainment of Walking in Rural Guatemalan Infants». The Journal of Nutrition. Vol. 134 No. 12.

Lutter, Chessa y Chaparro, Camila.2008. La desnutrición en lactantes y niños pequeños en América Latina y El Caribe: alcanzando los objetivos de desarrollo del milenio. Washington, D.C.: OPS

Organización de las Naciones Unidas para la Infancia. 2007. Estado mundial de la infancia 2008: Supervivencia Infantil. Nueva York: UNICEF. 
Paraje, Guillermo. 2008. Evolución de la desnutrición crónica infantil y su distribución socioeconómica en siete paises de América Latina y el Caribe. Santiago de Chile. CEPAL - Serie Políticas Sociales $N^{\circ} 140$.

Urteaga, Carmen y Pinheiro, Ana. 2003. «Investigación Alimentaria: Consideraciones Prácticas para Mejorar la Confiabilidad de los Datos». Rev. chil. nutr. Vol. 30, Nº 3. $235-242$.

Zarzalejo, Zulema y otros. 2001. «Hábitos de alimentación en niños desnutridos menores de dos años en una comunidad urbano marginal». AnVenezNutr. Vol.14, $\mathrm{N}^{\circ}$ 2. 60-69.

Fecha de recepción: 18 de octubre de 2016

Fecha de aceptación: 12 de diciembre de 2016

\section{Correspondencia}

Gina Mendoza Ramírez

Ginadmr1@hotmail.com 